\title{
A New Method to Calculate Induced Current of Thin Wire over Anisotropic Ground under HPM
}

\author{
Le Cao (iD) \\ School of Electric and Control Engineering, Xi'an University of Science and Technology, Xi'an, China \\ Correspondence should be addressed to Le Cao; xidian_caole@163.com
}

Received 25 October 2018; Revised 11 January 2019; Accepted 15 January 2019; Published 26 February 2019

Academic Editor: Ping Li

Copyright (C) 2019 Le Cao. This is an open access article distributed under the Creative Commons Attribution License, which permits unrestricted use, distribution, and reproduction in any medium, provided the original work is properly cited.

\begin{abstract}
The time response analysis of wire structures is often carried out in free space or isotropic half-space, but the real ground is usually layered and has anisotropic properties. In this paper, the induced current of a thin wire over layered anisotropic half-space under a high-power microwave (HPM) is calculated by using the time-domain integral equation (TDIE) method. The reflection coefficient of a layered anisotropic medium is obtained by the general transmitting matrix (GTM) method combined with Fourier transform. The variation of the induced current on the thin wire under different incident conditions is analyzed.
\end{abstract}

\section{Introduction}

A HPM pulse has a large peak power and strong directionality; it can lead to damage or cause failures in electronic devices. So this problem has a significant threat to an electronic system or equipment and has received increasing attention [1-3]. Once the thin wire is used to transmit energy in information telecommunication systems and electrical power systems in a high-power electromagnetic environment, the overcurrent and overvoltage will be formed on the device port through field-line coupling. As a result, the system performance is degraded or even permanently damaged. Therefore, the analysis of the near-field coupling of HPM on the thin wire has great research value for the protection of electronic and electrical systems.

The half-space problem is the widespread presence of wire structures working in the background, such as the transmission line over ground. Meanwhile, many half-space media have anisotropic properties, such as land covered by vegetation and the upper crust (especially in the sedimentary rock system). Numerical methods for solving these problems have been developed with different approaches. One is the transmission line method; four models have been proposed by Taylor et al., Agrawal et al., and Rachidi for describing the coupling of an external EM field to a line [4-6]. Generally, the transmission line method has computational errors when dealing with complex linear structures, such as models with bends. On the other hand, some frequency domain methods are used to solve this problem, such as the method of moment (MoM) [7, 8]. Frequency domain methods lead to accurate solutions but with an intensive use of computational resources, especially for the HPM pulse with very wide frequency bands; the time response calculation requires intensive computational resources. The same is true for algorithms based on the solution of the Sommerfeld problem $[9,10]$. Sommerfeld integration is extremely time-consuming, and it is even more difficult to deal with the multilayered half-space or anisotropic medium case. There are solutions based on approximations such as the reflection-coefficient method [11, 12], which is computationally faster, but as it assumes that the waves incident on the ground are plane waves, it presents losses of accuracy when the approximation is not valid [13]. Meanwhile, these problems can be solved by the finite-difference time-domain (FDTD) method [14-16], time-domain integral equation (TDIE) method [17-19], and discontinuous Galerkin time-domain (DGTD) method $[20,21]$. Most of these studies are carried out in free space or isotropic half-space because the reflected field of multilayered and even-layered anisotropic half-space is difficult to 
obtain. However, the existence of anisotropic media is more common in the natural environment. Therefore, we need a fast time-domain algorithm suitable for processing linear targets. Meanwhile, this algorithm is not limited by the medium in the lower half-space.

The TDIE method can obtain the transient response by one calculation and has significant advantages on dealing with linear objects. This is because Maxwell equations are solved just on the surface of objects, and there is no need to set up the absorbing boundary conditions. It means the broadband response can be calculated at any time, and this leads to a higher computational efficiency. Therefore, in this paper, a new method of combining TDIE with GTM is employed to analyze the transient response of the thin wire over layered anisotropic half-space. Considering the effect of anisotropic half-space, first, the reflected wave of the anisotropic half-space is obtained by the GTM method combined with Fourier transform. Then the two excitation sources conclude that the direct incident wave and reflected wave are introduced into the TDIE computational region. Finally, the induced current of the wire is obtained.

\section{Solution to the Coupling Problem of Thin Wire over Anisotropic Space}

The geometry of the problem to be discussed is shown in Figure 1. A single wire of radius $a$ and length $L$ is located at a height $H$ over a layered anisotropic ground plane. $\theta_{i}$ is the angle between the wave vector $\mathbf{k}$ and the normal vector of the interface. The incident plane is defined as the plane organized by $\mathbf{k}$ and the $z$-axis, and $\varphi$ is the angle between the incident plane and the $x o z$ plane. Suppose the incident wave electric field amplitude is $E_{0}$ and the polarization angle is $\alpha$, the electric field of the incident wave can be decomposed into a horizontal (to incident plane) component and a vertical component.

$$
\begin{aligned}
& E_{h}=E_{0} \sin \alpha, \\
& E_{v}=E_{0} \cos \alpha .
\end{aligned}
$$

Since the total tangential electric field on PEC equals zero, the current on the wire is determined by the total incident electric field, which is comprised of the incident field which is already given and the reflected field from the half-space. In this paper, the time-domain (TD) and frequency-domain (FD) methods are combined to solve the problem. First, the waveform of the reflected wave can be obtained by the Fourier transform and inverse Fourier transform. Then, both incident and reflected waves are introduced into the TDIE computational region. Finally, the total induced current can be obtained. The whole calculation procedure can be seen in Figure 2.

2.1. The Introduction of the TDIE Method. From Maxwell's equations in the time domain, we can use potential function A and scalar function $\phi$ to describe the electromagnetic field.

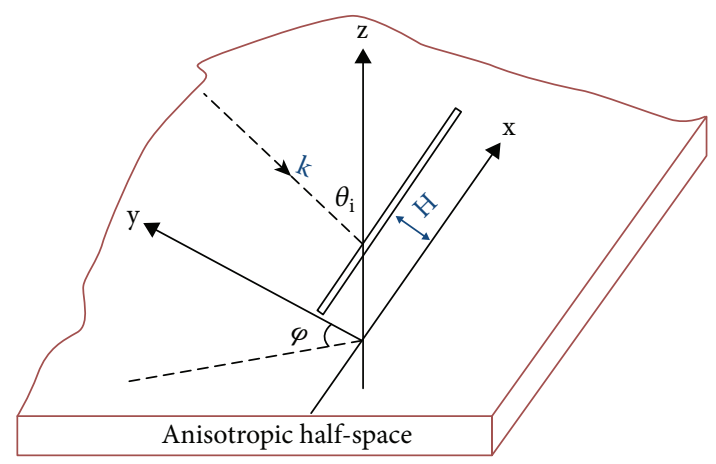

FIGURE 1: Thin wire over layered anisotropic half-space.

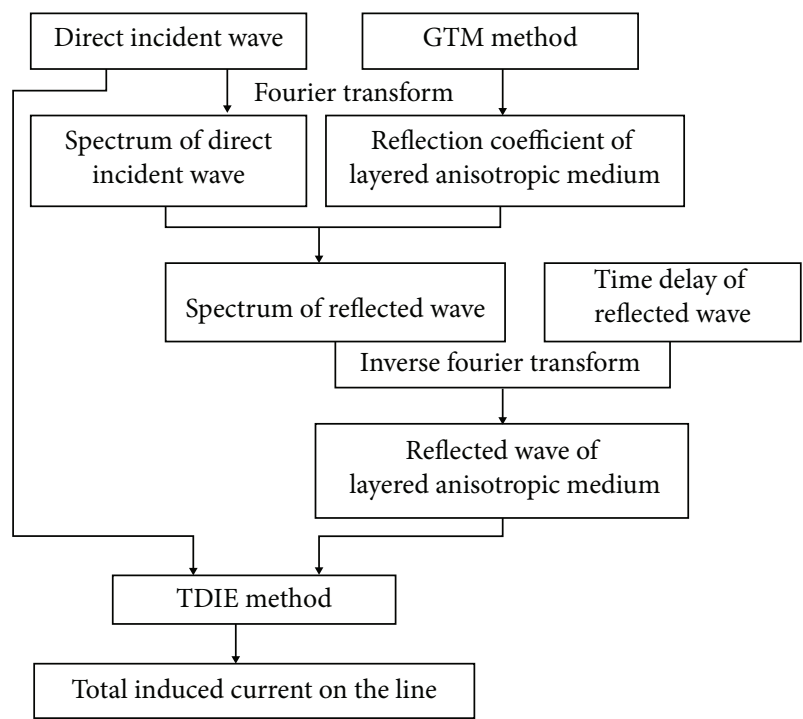

FIgURE 2: The flow diagram of the proposed method.

The wave equation satisfied by the potential function $\mathbf{A}$ under the Lorentz gauge condition is

$$
\begin{gathered}
\nabla^{2} \mathbf{A}-\frac{1}{c^{2}} \frac{\partial^{2} \mathbf{A}}{\partial t^{2}}=-\mu \mathbf{J}, \\
\nabla^{2} \phi-\frac{1}{c^{2}} \frac{\partial^{2} \phi}{\partial t^{2}}=-\frac{q}{\varepsilon}
\end{gathered}
$$

where $\mathbf{J}$ and $q$ represent the impressed source current density and source charge density, respectively. $\varepsilon$ and $\mu$ are the dielectric permittivity and the permeability, respectively. Meanwhile, these two equations have the same mathematical form with well-known solutions, given by

$$
\begin{aligned}
& \mathbf{A}(\mathbf{r}, t)=\frac{\mu}{4 \pi} \iiint_{V} \frac{\mathbf{J}\left(\mathbf{r}^{\prime}, t-R / c\right)}{R} \mathrm{~d} v^{\prime}, \\
& \phi(\mathbf{r}, t)=\frac{1}{4 \pi \varepsilon} \iiint_{V} \frac{q\left(\mathbf{r}^{\prime}, t-R / c\right)}{R} \mathrm{~d} v^{\prime},
\end{aligned}
$$

where $R=\left|\mathbf{r}-\mathbf{r}^{\prime}\right|$, and $\mathbf{r}$ and $\mathbf{r}^{\prime}$ represent the locations of the observation and source point, respectively. 
Consider a thin wire of length $2 L \mathrm{~m}$ and radius $a \mathrm{~m}$, located symmetrically along the $x$-axis as shown in Figure 3. The incident electric field induces current $I(x, t)$, which is a function of $x$ and $t$ only because of the thin wire approximation [22]. In this case, the vector potential is

$$
\mathbf{A}_{x}(x, t)=\frac{\mu}{4 \pi} \int_{x^{\prime}=-L}^{L} \frac{I\left(x^{\prime}, t-\left|x-x^{\prime}\right| / c\right)}{\sqrt{\left|x-x^{\prime}\right|^{2}+a^{2}}}
$$

and $\mathbf{A}$ satisfies the following equation:

$$
\frac{\partial^{2} A_{x}}{\partial x^{2}}-\left.\frac{1}{c^{2}} \frac{\partial^{2} A_{x}}{\partial t^{2}}\right|_{r=a}=-\left.\frac{1}{c^{2}} \frac{\partial E_{x}^{i}}{\partial t}\right|_{r=a}, \quad \text { for } x \in(0, L) .
$$

Now we divide the wire axis into $N+1$ equal subdomains of width $\Delta x$ with $N$ match points, $x_{1}, x_{2}, \ldots, x_{N}$, as shown in Figure 2. Note that the current is zero at both ends of the wire which implies that $I=0$ for $x=x_{0}=x_{N+1}$ at all times. Furthermore, the time axis is subdivided into small time intervals given by $\Delta t$ and denotes $t_{n}=n \Delta t, n=0,1,2, \ldots, \infty$.

First, a set of basis functions for expansion purposes is defined:

$$
f_{m}(x)= \begin{cases}1, & x_{m}-\frac{\Delta x}{2} \leq x \leq x_{m}+\frac{\Delta x}{2} \\ 0, & \text { otherwise }\end{cases}
$$

Using these expansion functions, we approximate the current $I$ as

$$
I(x, t) \approx \sum_{k=1}^{N} I_{k}(t) f_{k}(x)
$$

where $I_{k}(t)$ is the unknown coefficient at the $k$ th zone. Using equation (7), we can get

$$
\begin{aligned}
A_{m, n} & \equiv A\left(x_{m}, t_{n}\right) \\
& =\frac{\mu}{4 \pi} \int_{x^{\prime}=0}^{l} \frac{I\left(x^{\prime}, t_{n}-\left|x_{m}-x^{\prime}\right| / c\right)}{\sqrt{\left|x_{m}-x^{\prime}\right|^{2}+a^{2}}} \mathrm{~d} x^{\prime} \\
& \approx \frac{\mu}{4 \pi} \int_{x^{\prime}=0}^{l} \frac{\sum_{k=1}^{N} I_{k}\left(t_{n}-\left|x_{m}-x^{\prime}\right| / c\right) f_{k}\left(x^{\prime}\right)}{\sqrt{\left|x_{m}-x^{\prime}\right|^{2}+a^{2}}} \mathrm{~d} x^{\prime} \\
& =\sum_{k=1}^{N} I_{k}\left(t_{n}-\frac{\left|x_{m}-x_{k}\right|}{c}\right) \kappa_{m, k},
\end{aligned}
$$

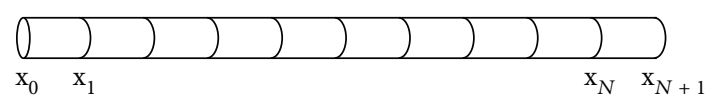

Figure 3: Wire divided into subdomains.

where

$$
\begin{aligned}
\kappa_{m, k}= & \frac{\mu}{4 \pi} \int_{x^{\prime}=x_{k}+\Delta x / 2}^{x_{k}+\Delta x / 2} \frac{\mathrm{d} x^{\prime}}{\sqrt{\left(x_{m}-x^{\prime}\right)^{2}+a^{2}}} \\
= & \frac{\mu}{4 \pi}\left\{\ln \left[\left(x_{m}-x_{k}+\frac{\Delta x}{2}\right)+\sqrt{\left(x_{m}-x_{k}+\frac{\Delta x}{2}\right)^{2}+a^{2}}\right]\right. \\
& \left.-\ln \left[\left(x_{m}-x_{k}-\frac{\Delta x}{2}\right)+\sqrt{\left(x_{m}-x_{k}-\frac{\Delta x}{2}\right)^{2}+a^{2}}\right]\right\} .
\end{aligned}
$$

We can also write equation (8) as

$$
A_{m, n}=I_{m, n} \kappa_{m, m}+A_{m, n}
$$

where

$$
A_{m, n}=\sum_{\substack{k=1 \\ k \neq m}}^{N} I_{k}\left(t_{n}-\frac{\left|x_{m}-x_{k}\right|}{c}\right) \kappa_{m, k} .
$$

Note that the bar on $A_{m, n}$ explicitly denotes that $k=m$ is omitted from the summation. When $k=m$, we have

$$
\kappa_{m, m}=\frac{\mu}{4 \pi} \ln \left[\frac{\sqrt{(\Delta x / 2)^{2}+a^{2}}+\Delta x / 2}{\sqrt{(\Delta x / 2)^{2}+a^{2}}-\Delta x / 2}\right] .
$$

Then we approximate the derivatives in equation (5) by central difference approximation to obtain

$$
\frac{A_{m+1, n}-2 A_{m, n}+A_{m-1, n}}{(\Delta x)^{2}}-\frac{A_{m, n+1}-2 A_{m, n}+A_{m, n-1}}{(c \Delta t)^{2}}=-F_{m, n} \text {, }
$$

where

$$
F_{m, n}=\frac{1}{c^{2}} \frac{\partial E_{x}^{i}\left(x_{m}, t_{n}\right)}{\partial t} .
$$

If we substitute equation (14) into equation (13), we can get

$$
\begin{aligned}
A_{m, n+1}= & 2 A_{m, n}-A_{m, n-1}+(\Delta t)^{2} F_{m, n} \\
& +\left(\frac{c \Delta t}{\Delta x}\right)^{2}\left[A_{m+1, n}-2 A_{m, n}+A_{m-1, n}\right]
\end{aligned}
$$


Finally, if we replace $n \longrightarrow n-1$ and use equation (10), we can write equation (15) as

$$
\begin{aligned}
I_{m, n} \kappa_{m, m}= & -A_{m, n}+2 A_{m, n-1}-A_{m, n-2}+(\Delta t)^{2} F_{m, n-1} \\
& +\left(\frac{c \Delta t}{\Delta x}\right)^{2}\left[A_{m+1, n-1}-2 A_{m, n-1}+A_{m-1, n-1}\right] .
\end{aligned}
$$

The algorithm may be started by assuming $I_{m, 0}=I_{m, 1}=0$ and calculating $I_{m, 2}$ using equation (16). Once $I_{m, 2}$ is obtained, we can proceed to calculate $I_{m, 3}$. This procedure can be continued to calculate the current at the following time instants. Therefore, this method is popularly known as the marching on in time (MOT) method and is quite efficient for computing the transient response from a thin wire. An important advantage of this method is that no matrix inversion is required as long as we choose $\Delta t \leq(\Delta x) / c$.

For the half-space problem, the $F_{m, n}$ must conclude the incident wave and reflected wave, so it can be rewritten as

$$
F_{m, n}=\frac{1}{c^{2}} \frac{\partial E_{x}^{i}\left(x_{m}, t_{n}\right)}{\partial t}+\frac{1}{c^{2}} \frac{\partial E_{x}^{r}\left(x_{m}, t_{n}\right)}{\partial t}
$$

where $E^{r}$ is the reflected electric field of half-space interface.

2.2. Calculation of Reflection Coefficients. The basic theories of waves reflected by layered anisotropic media are summarized by Chew [23]. In a homogeneous, anisotropic medium, there exist two types of waves which are type I and type II. The general reflection coefficient $\overline{\bar{R}}$ and transmission coefficient $\overline{\bar{T}}$ of layered anisotropic media are defined as

$$
\begin{aligned}
& \overline{\bar{R}}=\left[\begin{array}{ll}
R_{\mathrm{I}, \mathrm{I}} & R_{\mathrm{I}, \mathrm{II}} \\
R_{\mathrm{II}, \mathrm{I}} & R_{\mathrm{II}, \mathrm{II}}
\end{array}\right], \\
& \overline{\bar{T}}=\left[\begin{array}{cc}
T_{\mathrm{I}, \mathrm{I}} & T_{\mathrm{I}, \mathrm{II}} \\
T_{\mathrm{II}, \mathrm{I}} & T_{\mathrm{II}, \mathrm{II}}
\end{array}\right] .
\end{aligned}
$$

In general, these two types of waves are decoupled in a homogeneous medium. As shown in Figure 4, in the presence of a planar interface, however, they will be coupled to each other at the interface. In other words, a type I wave may generate transmitted and reflected waves of type I and type II. $R_{\mathrm{I}, \mathrm{II}}\left(R_{\mathrm{II}, \mathrm{I}}\right)$ in equation (18) represents the reflection coefficient with type I(II) wave incidents but the reflection is a type II(I) wave. If a medium is isotropic instead, the type I and type II waves reduce to TE and TM waves.

For anisotropic medium, both the relative permittivity $\overline{\bar{\varepsilon}}=\left[\varepsilon_{i j}\right]$ and the permeability $\overline{\bar{\mu}}=\left[\mu_{i j}\right]$ are a second-order tensor. For source-free, anisotropic media, we have

$$
\begin{aligned}
\nabla \times \mathbf{E} & =-\mathrm{i} \omega \mu \cdot \mathbf{H}, \\
\nabla \times \mathbf{H} & =\mathrm{i} \omega \varepsilon \cdot \mathbf{E} .
\end{aligned}
$$

If we let the $z$-axis be perpendicular to the media interface, the operators $\nabla, \mathbf{E}, \mathbf{H}, \varepsilon$, and $\mu$ can be decomposed. Then

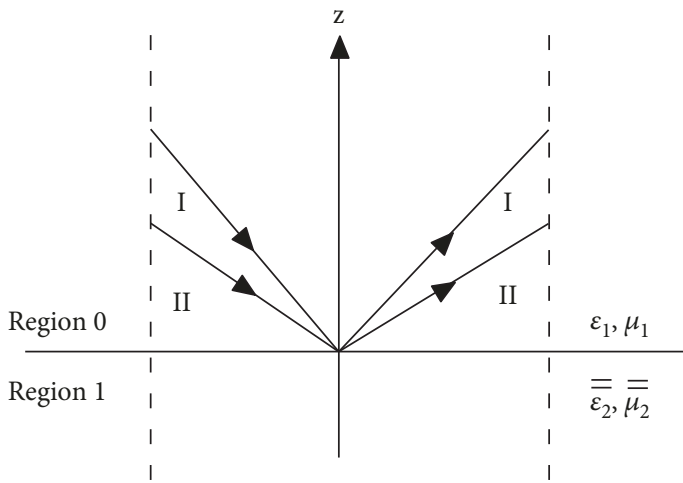

FIgURE 4: The reflection of the anisotropic interface.

from the phase matching condition, the state equation can be obtained.

$$
\frac{d}{d z} \mathbf{S}=\mathbf{C} \cdot \mathbf{S}
$$

The general solution of this equation is

$$
\mathbf{S}(z)=a \cdot \exp (i \lambda z) \cdot \mathbf{B}
$$

The propagation properties of plane waves in the medium can be expressed as $\bar{V}(z)=\mathbf{P}\left(z, z^{\prime}\right) \cdot \bar{V}\left(z^{\prime}\right) . \mathbf{P}\left(z, z^{\prime}\right)$ is known as the propagator matrix or the transition matrix. It relates the state vectors that describe the fields at two different locations $z$ and $z^{\prime}$. For $n$-layer media shown in Figure 5, the propagator matrix of the entire media plate is equal to the product of the propagator matrix of each layer, that is,

$$
\mathbf{P}\left(z_{n}, z_{0}\right)=\prod_{l=n}^{1} \mathbf{P}_{l}\left(z_{l}, z_{l-1}\right) .
$$

The region $n+1$ only exists in the transmitted wave:

$$
\left[\begin{array}{c}
\overline{\overline{0}} \\
\overline{\bar{T}}
\end{array}\right]=\left(\overline{\bar{a}}^{n+1}\right)^{-1} \cdot \overline{\bar{P}}\left(z_{n}, z_{0}\right) \cdot \overline{\bar{a}}^{0} \cdot\left[\begin{array}{c}
\overline{\bar{R}} \\
\overline{\bar{I}}
\end{array}\right] .
$$

So after finding the eigenmatrix $\overline{\bar{a}}^{0}$ and $\overline{\bar{a}}^{n+1}, \overline{\bar{R}}$ and $\overline{\bar{T}}$ can be solved.

\section{Numerical Results}

3.1. Algorithm Verification. Example 1 verifies the correctness of the reflection coefficient of the single-layered anisotropic medium. The reflection coefficients of the isotropicanisotropic interface have analytic expressions in some special cases [24]. Figure 6 shows the comparison between the proposed method and the analytical method; it can be seen that the results of the proposed method and the analytic method are in good agreement.

To demonstrate the correctness of the proposed method, we consider the reflected wave of the single-layered anisotropic medium. The discrete grid $\delta$ in FDTD is $0.04 \mathrm{~cm}$ and 


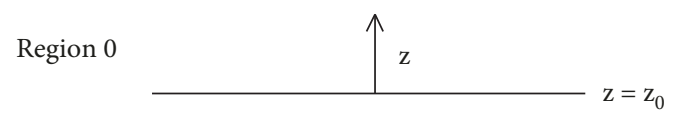

Region 1

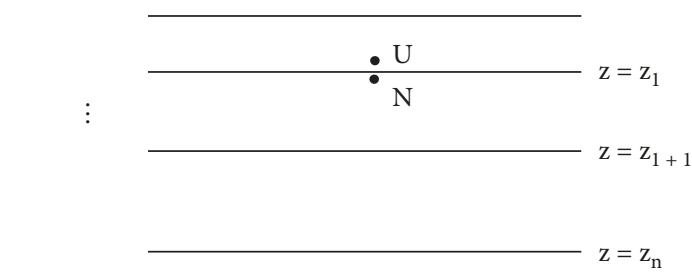

Region $\mathrm{n}+1$

Figure 5: Layered anisotropic medium.

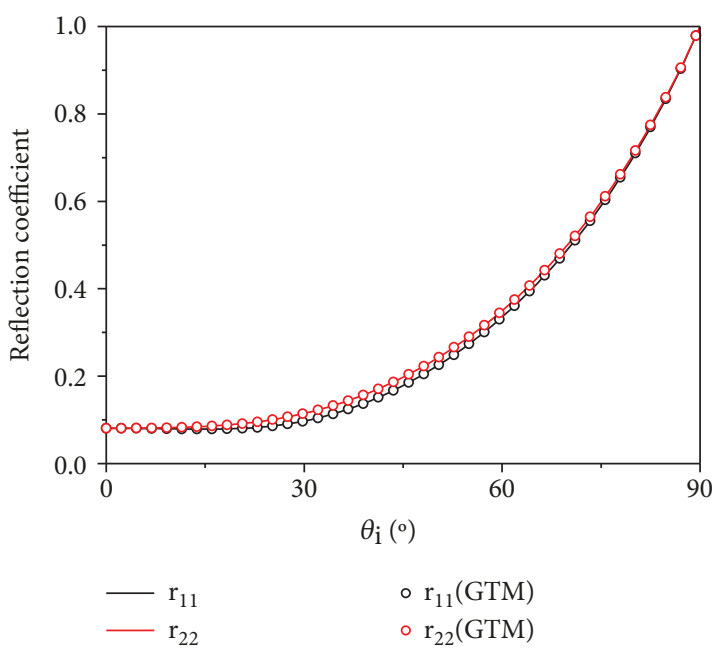

Figure 6: The reflection coefficients of the analytic method and the GTM method.

the absorbing boundary is CPML. The incident wave is HEMP which can be described as

$$
E=k E_{0}\left[\exp \left(-\alpha t^{\prime}\right)-\exp \left(-\beta t^{\prime}\right)\right]
$$

where $k=1.05, \alpha=4.0 \times 10^{6} \mathrm{~s}^{-1}$, and $\beta=4.76 \times 10^{8} \mathrm{~s}^{-1}$.

The anisotropic half-space

$$
\begin{aligned}
\varepsilon_{r} & =\operatorname{diag}[5.6,30,5.6], \\
\sigma & =\operatorname{diag}[0.3,0.025,0.3] \mathrm{S} / \mathrm{m} .
\end{aligned}
$$

The results of the one-dimensional Maxwell's equation method [25] and the proposed method are shown in Figure 7. It can be seen that they are in good agreement.

Since the literature related to the cable coupling problem above the anisotropic half-space is relatively rare, in order to verify the correctness of proposed algorithm, an example of the isotropic medium is given. Figure 8 shows the induced current at the center of the thin wire of dimensions $L=1 \mathrm{~m}$ and $a=2 \mathrm{~mm}$ horizontally located at the height $h=0.25 \mathrm{~m}$ over lossy ground $\left(\varepsilon_{r}=10\right)$.

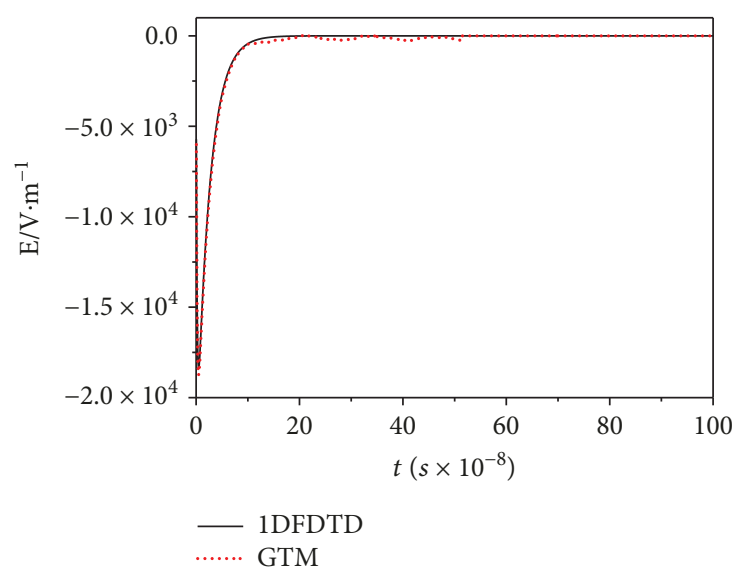

Figure 7: The reflected wave of FDTD and Fourier transform method.

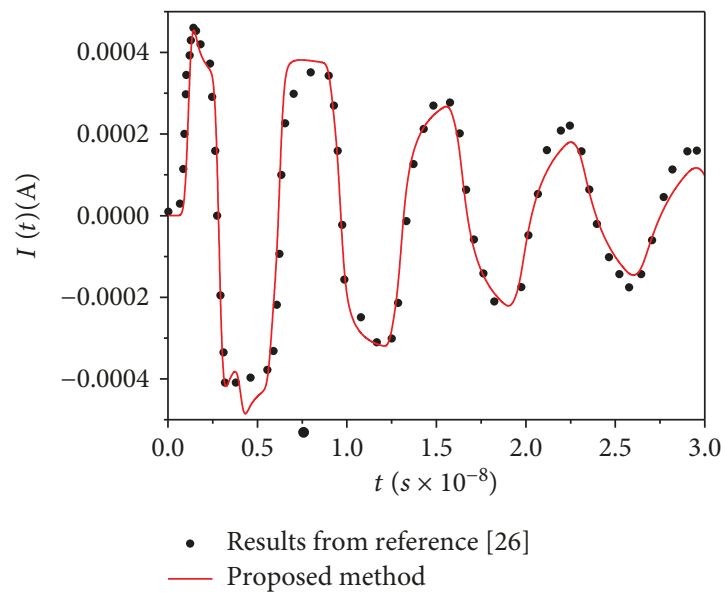

FIgURE 8: Induced current of the thin wire excited by pulse over lossy ground [26].

Often, the induced current is associated with many factors, such as the length of the thin wire, the incident angle, and the height between the thin wire and the half-space interface. The variation of the induced current under different conditions is discussed in the following section.

3.2. Effect of Thin-Wire Length on Induced Current. Figure 9 shows the induced current at the center of the thin wire with different lengths located at $H=3 \mathrm{~m}$ over the anisotropic halfspace $\left(\varepsilon_{r}=\operatorname{diag}[5.6,5.6,30], \sigma=\operatorname{diag}[0.3,0.025,0.3] \mathrm{S} / \mathrm{m}\right)$. The incident wave is a UWB pulse with incident angles of $\theta_{i}=30^{\circ}$ and a polarization angle of $\alpha=30^{\circ}$.

As can be seen from Figure 9, the magnitude of the induced current is closely related to the length of the thin wire. As the length of the thin wire increases, magnitude of the induced current increases and the oscillation period becomes longer. From Figure 10, we can see that for thin wires of tens of meters or more, its frequency response to the UWB pulse is typically less than $30 \mathrm{MHz}$ in this condition. 


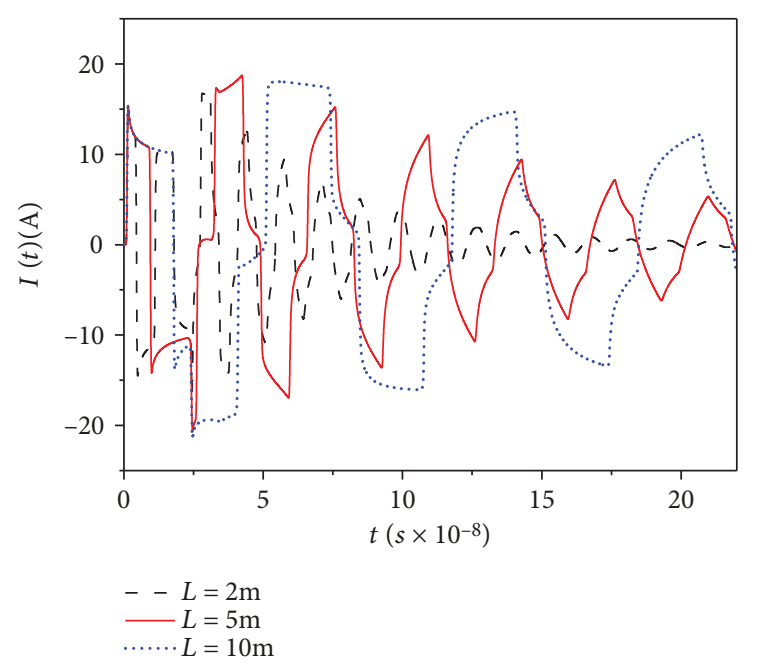

FIGURE 9: The induced current at the center of the thin wire with different lengths.

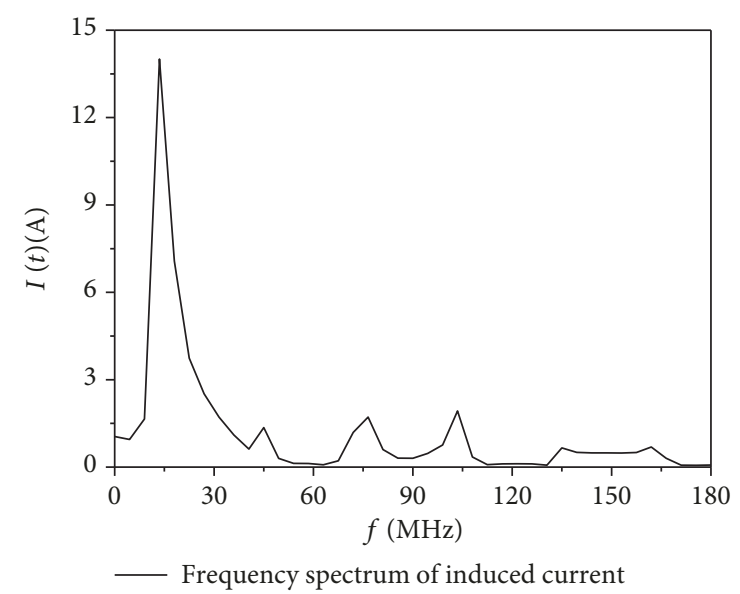

Figure 10: The frequency spectrum of induced current when $L=10 \mathrm{~m}$.

3.3. Effect of Incident Angle on Induced Current. The reflection coefficient is directly related to the incident angle. In order to facilitate the discussion, we take $f=35.16 \mathrm{GHz}$ as an example to show the variation of the reflection coefficient of a uniaxially anisotropic medium with an increasing incident angle. From Figure 11, it can be seen that for an electrically uniaxially anisotropic medium, $r_{12}=r_{22}=0, r_{11}$ increases with the incident angle, and $r_{22}$ decreases with the incident angle and then increases, reaching a minimum at the Brewster angle.

Figure 12 show the variation of the induced current at different incident angles for horizontal $\alpha=0^{\circ}$ and vertical $\alpha=90^{\circ}$ polarization incident waves.

For vertical polarized incident waves, first, the induced current increases as the incident angle increases. Since the vertically polarized incident wave has no horizontal component and $r_{11}$ increases with the increase of the incident wave, the induced current of the thin wire increases with the incident angle. Second, the delay time of the reflected wave

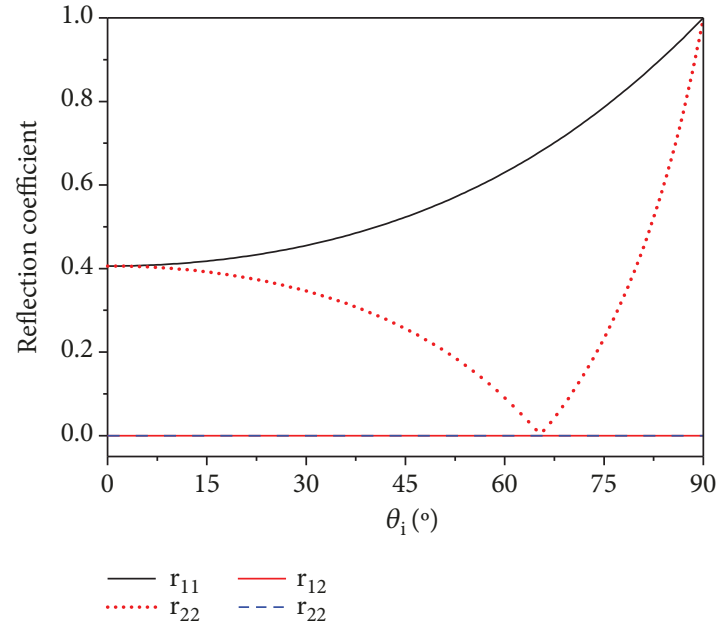

FIgURE 11: The variation of the reflection coefficient of incident angle.

arriving at the thin wire (compared to the direct incident wave) increases as the incident angle increases. When $\theta_{i}=80^{\circ}$, the reflected wave delay time is longer, so the variation of the induced current is completely consistent with the free space before $t=\left(11 \times 10^{-8}\right) \mathrm{s}$. After the reflected wave reaches the thin wire, the induced current increases. However, for the horizontal polarized incident wave, the induced current decreases first and then increases with the incident angle. This is because there is no vertical component of the incident wave, and the corresponding horizontal reflection coefficient $r_{22}$ will decrease first and then increase with the incident angle. However, the delay time of the reflected wave arriving at the thin wire (as compared with the direct incident wave) still increases with the incident angle.

3.4. Effect of H on Induced Current. The overhead thin wire is excited by the UWB pulse with the incident angle $\theta_{i}=30^{\circ}$ and polarization angle $\alpha=30^{\circ}$. Figure 13 shows the variation of the induced current with time of overhead thin wire at different heights. As can be seen from the figure, the magnitude of the induced current has a significant change relationship with the height between the thin wire and the interface. The induced current of the overhead cable $(H=3 \mathrm{~m})$ is significantly greater than that of the ground cable $(H=0.03 \mathrm{~m})$.

\section{Conclusion}

In this paper, the induced current of the thin wire over the anisotropic half-space is calculated by using the TDIE method combined with Fourier transform. The reflection coefficient of the anisotropic medium is obtained by the GTM method. The variation of the midpoint which induced current on the thin wire under different conditions can be summarized as follows:

(1) As the length increases, the induced current on the thin wire increases and the oscillation period becomes longer. For thin wires of tens of meters or 


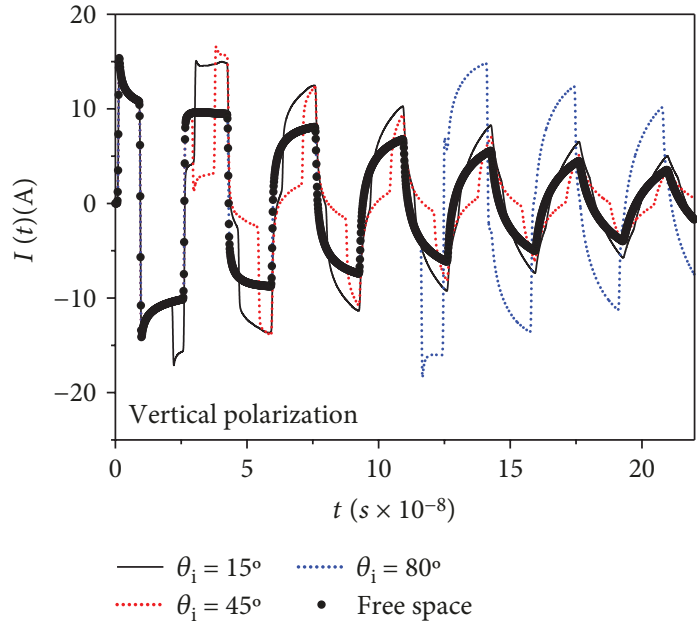

(a) Vertical polarization

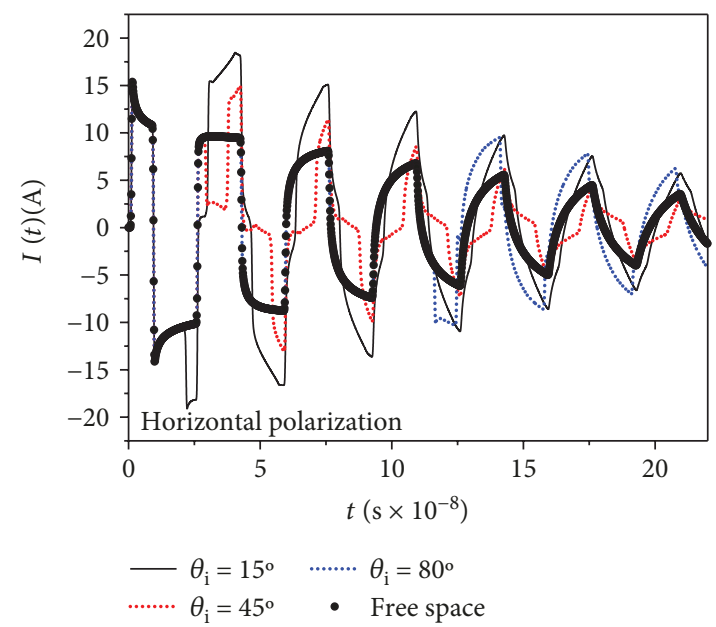

(b) Horizontal polarization

FIGURE 12: The frequency spectrum of induced current when $L=10 \mathrm{~m}$.

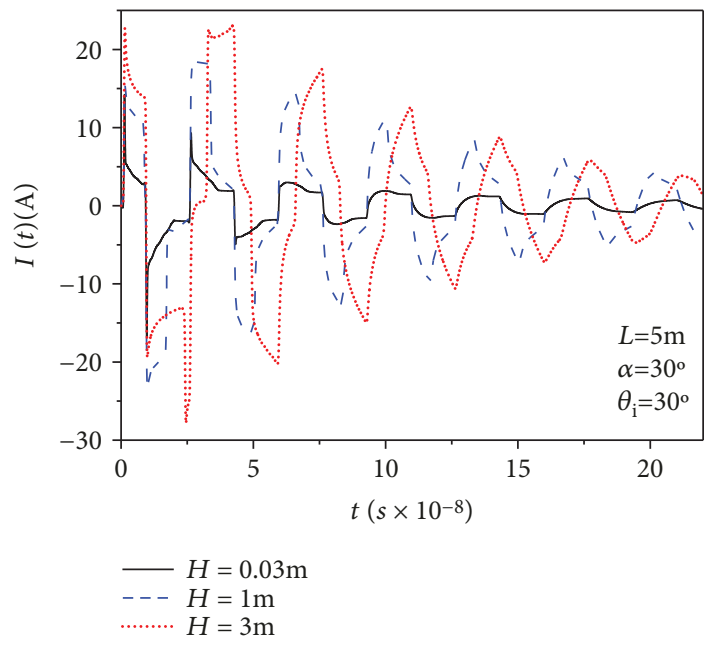

FIGURE 13: The comparison of induced current between isotropic and anisotropic media.

more in length, the frequency response of the UWB pulse is generally below $30 \mathrm{MHz}$

(2) For vertically polarized incident waves, the induced current of the thin wire increases with the incident angle. For horizontally polarized incident waves, the induced current of the thin wire increases first and then decreases with the incident angle.

(3) The magnitude of the induced current on the overhead thin wire increases with the heights between the thin-wire and the half-space interface. That is, the induced current on the overhead cable is significantly greater than the ground cable.

\section{Data Availability}

The data used to support the findings of this study are available from the corresponding author upon request.

\section{Conflicts of Interest}

The author declares that she has no conflicts of interest.

\section{Acknowledgments}

The project was supported by the Shaanxi Provincial Education Department (Program No. 17JK0496).

\section{References}

[1] V. N. Greetsai, A. H. Kozlovsky, V. M. Kuvshinnikov et al., "Response of long lines to nuclear high-altitude electromagnetic pulse (HEMP)," IEEE Transactions on Electromagnetic Compatibility, vol. 40, no. 4, pp. 348-354, 1998.

[2] Y. Z. Xie, J. Guo, and G. Canavero, "Analytic iterative solution of electromagnetic pulse coupling to multiconductor transmission lines," IEEE Transactions on Electromagnetic Compatibility, vol. 55, no. 3, pp. 451-466, 2013.

[3] S. Peng, C. Yuan, T. Shu, and X. Zhao, "Linearly polarised radial line slot antenna for high-power microwave application," IET Microwaves, Antennas \& Propagation, vol. 11, no. 5, pp. 680-684, 2017.

[4] C. Taylor, R. Satterwhite, and C. Harrison, "The response of a terminated two-wire transmission line excited by a nonuniform electromagnetic field," IEEE Transactions on Antennas and Propagation, vol. 13, no. 6, pp. 987-989, 1965.

[5] A. Agrawal, H. Price, and S. Gurbaxani, "Transient response of multiconductor transmission lines excited by a nonuniform electromagnetic field," IEEE Transactions on Electromagnetic Compatibility, vol. EMC-22, no. 2, pp. 119-129, 1980.

[6] F. Rachidi, "A review of field-to-transmission line coupling models with special emphasis to lightning-induced voltages on overhead lines," IEEE Transactions on Electromagnetic Compatibility, vol. 54, no. 4, pp. 898-911, 2012.

[7] P. Parhami and R. Mittra, "Wire antennas over a lossy halfspace," IEEE Transactions on Antennas and Propagation, vol. 28 , no. 3, pp. 397-403, 1980. 
[8] S. Vitebskiy and L. Carin, "Moment-method modeling of short-pulse scattering from and the resonances of a wire buried inside a lossy, dispersive half-space," IEEE Transactions on Antennas and Propagation, vol. 43, no. 11, pp. 13031312, 1995.

[9] G. Burke and E. Miller, "Modeling antennas near to and penetrating a lossy interface," IEEE Transactions on Antennas and Propagation, vol. 32, no. 10, pp. 1040-1049, 1984.

[10] T. J. Cui and W. C. Chew, "Accurate model of arbitrary wire antennas in free space, above or inside ground," IEEE Transactions on Antennas and Propagation, vol. 48, no. 4, pp. 482-493, 2000.

[11] E. J. Rothwell, "Efficient computation of the time-domain TM plane-wave reflection coefficient," IEEE Transactions on Antennas and Propagation, vol. 53, no. 10, pp. 34173419, 2005.

[12] M. Fernández Pantoja, A. G. Yarovoy, A. Rubio Bretones, and S. González García, "Time domain analysis of thin-wire antennas over lossy ground using the reflection-coefficient approximation," Radio Science, vol. 44, no. 6, 2009.

[13] A. Karwowski and K. A. Michalski, "A comparative numerical study of several techniques for modeling a horizontal wire antenna over a lossy half-space," Radio Science, vol. 22, no. 6 , pp. 922-928, 1987.

[14] Z. X. Liang, H. Xie, Y. Guo et al., "Improved hybrid leapfrog ADI-FDTD method for simulating near-field coupling effects among multiple thin wire monopole antennas on a complex platform," IEEE Transactions on Electromagnetic Compatibility, vol. 59, no. 2, pp. 618-626, 2017.

[15] K. Niu, Z. Huang, M. Li, and X. Wu, "Optimization of the artificially anisotropic parameters in WCS-FDTD method for reducing numerical dispersion," IEEE Transactions on Antennas and Propagation, vol. 65, no. 12, pp. 7389-7394, 2017.

[16] A. Tatematsu, "A technique for representing lossy thin wires and coaxial cables for FDTD-based surge simulations," IEEE Transactions on Electromagnetic Compatibility, vol. 60, no. 3, pp. 705-715, 2018.

[17] S. B. Sayed, H. A. Ulku, and H. Bagci, "A stable marching on-in-time scheme for solving the time-domain electric field volume integral equation on high-contrast scatterers," IEEE Transactions on Antennas and Propagation, vol. 63, no. 7, pp. 3098-3110, 2015.

[18] H. H. Zhang, L. J. Jiang, and H. M. Yao, "Embedding the behavior macromodel into TDIE for transient field-circuit simulations," IEEE Transactions on Antennas and Propagation, vol. 64, no. 7, pp. 3233-3238, 2016.

[19] H. H. Zhang, Z. H. Fan, and R. S. Chen, "Marching-on-indegree solver of time-domain finite element-boundary integral method for transient electromagnetic analysis," IEEE Transactions on Antennas and Propagation, vol. 62, no. 1, pp. 319-326, 2014.

[20] G. S. Cheng, D. Z. Ding, and R. S. Chen, "An efficient fast algorithm for accelerating the time-domain integral equation discontinuous Galerkin method," IEEE Transactions on Antennas and Propagation, vol. 65, no. 9, pp. 4919-4924, 2017.

[21] P. Li, Y. Shi, L. J. Jiang, and H. Bagci, "Transient analysis of lumped circuit networks loaded thin wires by DGTD method," IEEE Transactions on Antennas and Propagation, vol. 64, no. 6, pp. 2358-2369, 2016.

[22] S. M. Rao, Transient Electromagnetics, Academic Press, New York, NY, USA, 1999.
[23] W. C. Chew, Waves and Fields in Inhomogeneous Media, Van Nostrand Reinhold, New York, NY, USA, 1990.

[24] H. C. Chen, Theory of Electromagnetic Waves: A Coordinate Free Approach, McGraw-Hill, New York, NY, USA, 1983.

[25] Y. N. Jiang, D. B. Ge, and S. J. Ding, "Analysis of TF-SF boundary for 2D-FDTD with plane $p$-wave propagation in layered dispersive and lossy media," Progress In Electromagnetics Research, vol. 83, pp. 157-172, 2008.

[26] D. Poljak and V. Roje, "Time domain calculation of the parameters of thin wire antennas and scatterers in a half-space configuration," IEE Proceedings - Microwaves, Antennas and Propagation, vol. 145, no. 1, p. 57, 1998. 


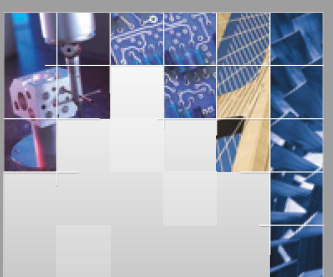

\section{Enfincering}
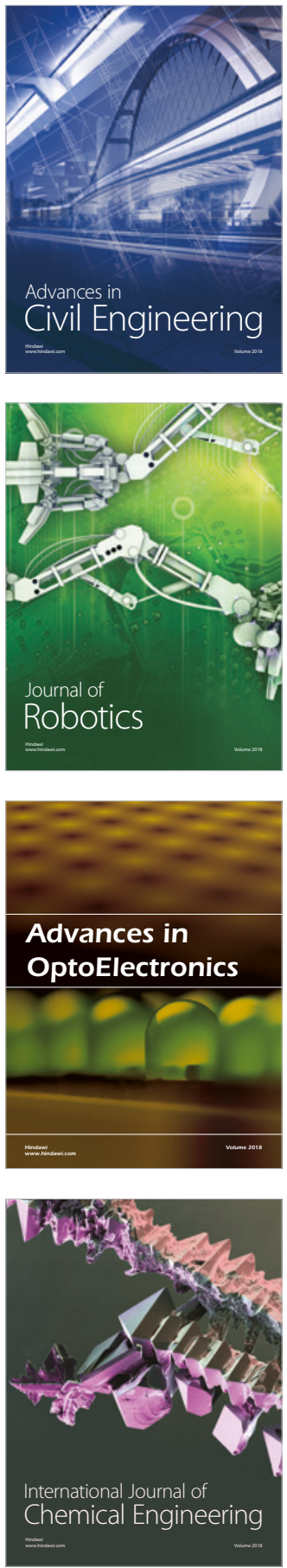

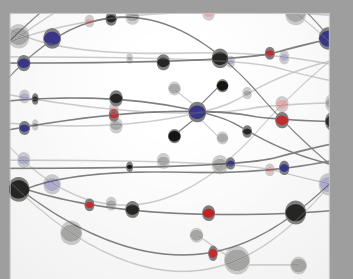

\section{Rotating \\ Machinery}

The Scientific World Journal

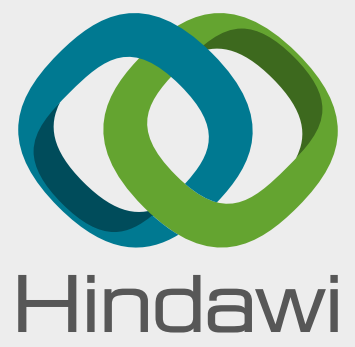

Submit your manuscripts at

www.hindawi.com
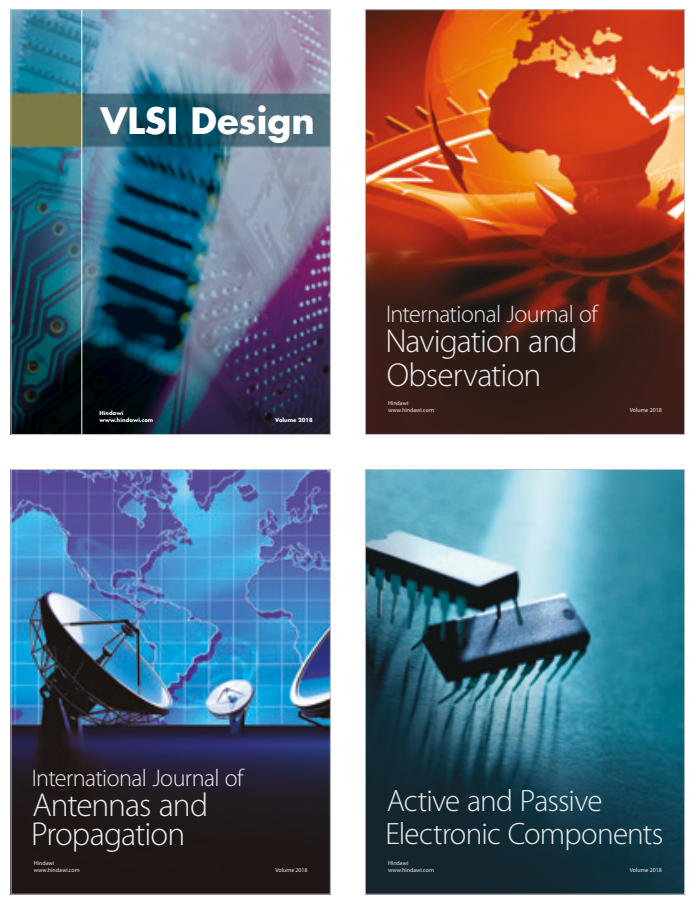
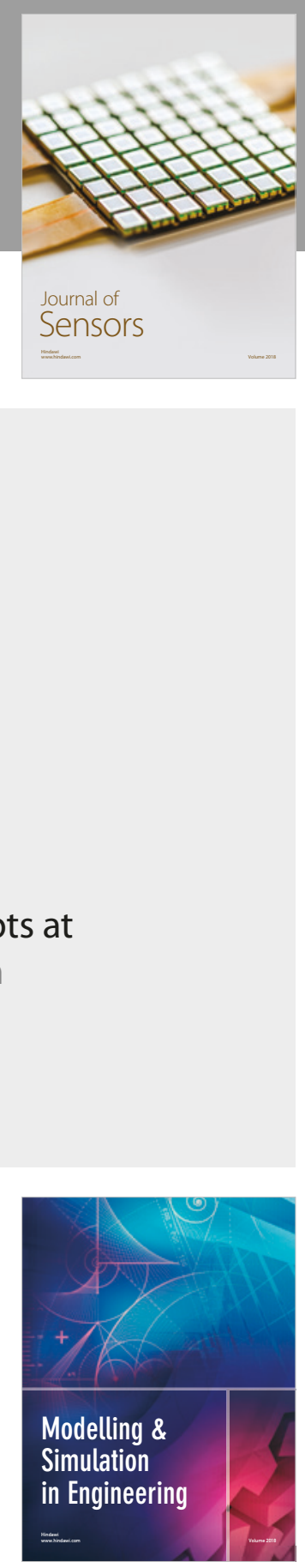

\section{Advances \\ Multimedia}
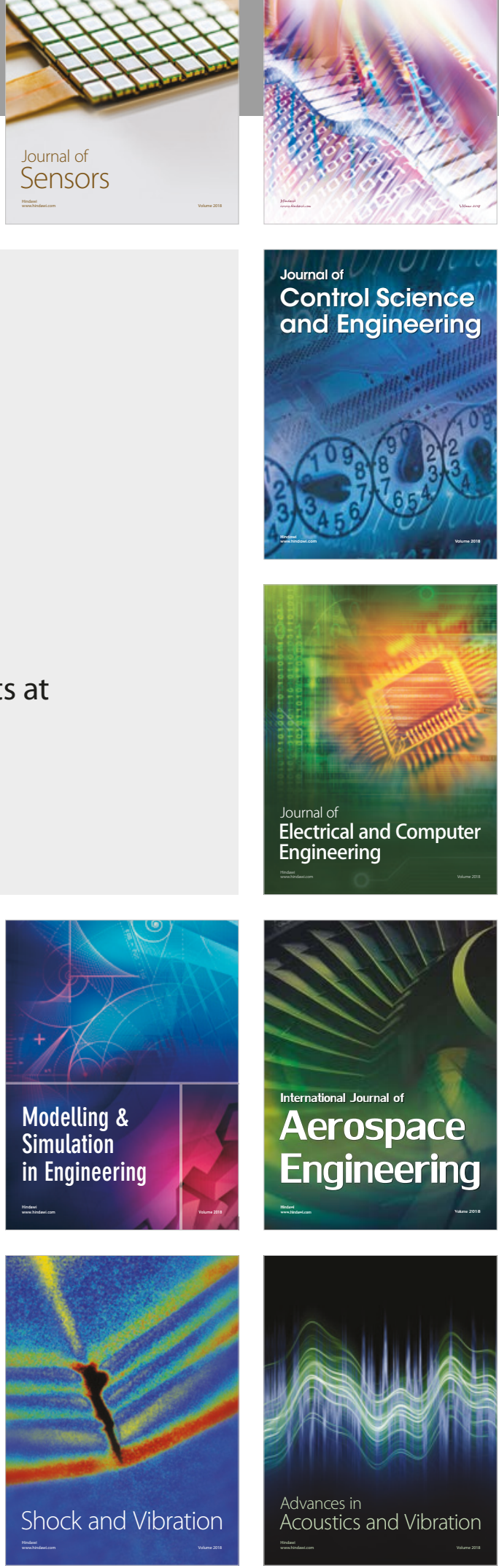